\title{
Dualities for Ising Networks
}

\author{
Yu-tin Huang, ${ }^{1,2}$ Chia-Kai Kuo, ${ }^{1}$ and Congkao $\mathrm{Wen}^{3}$ \\ ${ }^{1}$ Department of Physics and Astronomy, National Taiwan University, Taipei 10617, Taiwan \\ ${ }^{2}$ Physics Division, National Center for Theoretical Sciences, National Tsing-Hua University, \\ No.101, Section 2, Kuang-Fu Road, Hsinchu, Taiwan \\ ${ }^{3}$ Centre for Research in String Theory, School of Physics and Astronomy Queen Mary University of London, \\ Mile End Road, London E1 4NS, United Kingdom
}

(Received 11 September 2018; revised manuscript received 10 November 2018; published 21 December 2018)

\begin{abstract}
In this Letter, we study the equivalence between planar Ising networks and cells in the positive orthogonal Grassmannian. We present a microscopic construction based on amalgamation, which establishes the correspondence for any planar Ising network. The equivalence allows us to introduce two recursive methods for computing correlators of Ising networks. The first is based on duality moves, which generate networks belonging to the same cell in the Grassmannian. This leads to fractal lattices where the recursion formulas become the exact renormalization group equations of the effective couplings. The second, we use an amalgamation in which each iteration doubles the size of the seed lattice. This leads to an efficient way of computing the correlator where the complexity scales logarithmically with respect to the number of spin sites.
\end{abstract}

DOI: 10.1103/PhysRevLett.121.251604

Introduction.-In recent years there has been a fascinating interplay between the physics of observables in quantum field theories and geometries in mathematics. Consistency conditions of the observables, arising from fundamental principles of unitarity, locality, and symmetries, are often connected to the defining properties of certain mathematical objects. For instance, scattering amplitudes of gauge theories are connected to a positive Grassmannian [1,2] and further into the Amplituhedron [3], and couplings of higherdimensional operators in effective field theories, or fourpoint functions of a conformal field theory, are bounded by cyclic polytopes [4]. In each case, the mathematical object of interest has an intrinsic definition that does not make any reference to physics. In other words the physical principles become emergent from the mathematical properties.

Recently, a fascinating new connection was revealed by Galashin and Pylyavskyy [5]. The observables in question are correlators of two-dimensional (2D) planar Ising networks, which were shown to be equivalent to cells in a positive orthogonal Grassmannian. The latter was known to describe amplitudes of 3D supersymmetric Chern-Simon matter theories $[2,6]$.

In relating amplitudes to a positive Grassmannian, the physical principle underlying the equivalence is factorization:

Published by the American Physical Society under the terms of the Creative Commons Attribution 4.0 International license. Further distribution of this work must maintain attribution to the author(s) and the published article's title, journal citation, and DOI. Funded by SCOAP ${ }^{3}$. start with amplitudes of the fewest particles for which the correspondence is evident, and higher multiplicity amplitudes can be constructed via factorization, which is a positivity preserving operation. In this Letter, we identify the corresponding principle for Ising correlators: with amalgamation, under which two of (adjacent) external spin sites are identified, the correlation function of the new network can be written as a nonlinear function of the former. In terms of Grassmannians, the map linearizes and manifestly preserves positivity.

The correspondence allows us to introduce recursive methods to compute correlators with a large number of spin-sites by directly constructing a Grassmannian. First, utilizing the fact that duality moves in a Grassmannian reflect dual relations between networks, we introduce iterative duality moves to construct self-similar lattices. As the moves are equipped with maps between the couplings of the two lattices, the duality map can be interpreted as an exact renormalization group $(\mathrm{RG})$ equation. Alternatively, one can employ amalgamation in the Grassmannian to construct lattices that are self-repeating in one direction for an arbitrary length. In this case, since the complexity of computing the move is agnostic to the underlying network, it reduces the scale of the complexity of a lattice with $N$ sites to $\sim \log N$.

Mapping Ising network to cells of $\mathrm{OG}_{\geq 0}(n, 2 n)$. - For a general Ising network, the two-point function is defined as [7]

$$
\left\langle\sigma_{i} \sigma_{j}\right\rangle=\frac{\sum_{\sigma_{a} \in\{ \pm 1\}} \sigma_{i} \sigma_{j} P\left(J_{a b}\right)}{\sum_{\sigma_{a} \in\{ \pm 1\}} P\left(J_{a b}\right)}, \quad P\left(J_{a b}\right)=\prod_{a, b \in\{E\}} e^{J_{a b} \sigma_{a} \sigma_{b}}
$$


where $\sigma_{i}= \pm 1$ represent spin sites, $E$ is the set of edges and $J_{a b} \geq 0$ is the coupling constant connecting sites $a$ and $b$. Intuitively, since we are considering ferromagnetic couplings, we expect the correlator to be non-negative. However, as a sum with alternating signs, its positivity is not obvious. Remarkably, as proven in [8], not only is Eq. (1) positive, all minors of a $n \times n$ unit (with 1 in diagonal) symmetric matrix $\left\langle\sigma_{i} \sigma_{j}\right\rangle$ are positive (with definite signs). This is referred to as total positivity.

The unit symmetric matrix can be naturally embedded in a $n \times 2 n$ matrix $m_{i j}$ with the following map [5]:

$i \neq j: m_{i, 2 j-1}=-m_{i, 2 j}=\operatorname{sgn}[i-j](-)^{i+j}\left\langle\sigma_{i} \sigma_{j}\right\rangle$,

$i=j: m_{i, 2 i-1}=m_{i, 2 i}=1$.

The rows of the $n \times 2 n$ matrix are mutually null vectors (with alternating metric [2]), termed orthogonal Grassmannian $\mathrm{OG}_{n}$. Correlators can be recovered by the inverse map,

$$
\left\langle\sigma_{i} \sigma_{j}\right\rangle=\frac{\sum_{I \in \varepsilon(\{i, j\})} \Delta_{I}}{\sum_{I \in \varepsilon(\{\varnothing\})} \Delta_{I}} .
$$

Here, $\Delta_{I}$ denotes $n \times n$ minors of $\mathrm{OG}_{n}$, and $\varepsilon(\{S\})$ represents $n$-element subsets such that for each $i$, $I \bigcap(2 i-1,2 i)$ even times if and only if $i \in S$. As the simplest example, consider the network with spin sites connected by an edge $J$, the corresponding $\mathrm{OG}_{2}$ is

$$
\left(\begin{array}{cccc}
1 & s(J) & 0 & -c(J) \\
0 & c(J) & 1 & s(J)
\end{array}\right)
$$

where

$$
s(J)=\frac{2}{e^{2 J}+e^{-2 J}}, \quad c(J)=\frac{e^{2 J}-e^{-2 J}}{e^{2 J}+e^{-2 J}} .
$$

With $\Delta_{12}=c(J), \Delta_{13}=1, \Delta_{14}=s(J)$, using Eq. (3) we indeed recover the two-point function.

Note that, any unit symmetric matrix can be embedded in an $\mathrm{OG}_{n}$, and what is special for correlators of $2 \mathrm{D}$ planar Ising networks is that the corresponding $\mathrm{OG}_{n}$ is positive $\left(\mathrm{OG}_{\geq 0, n}\right)$ [5], i.e., all ordered $\Delta_{I} \geq 0$. Via Eq. (3), the total positivity of $\left\langle\sigma_{i} \sigma_{j}\right\rangle$ can be inferred from the $\mathrm{OG}_{\geq 0, n}$.

The microscopic derivation of the correspondence.-We will establish the correspondence by showing that any Ising network can be constructed from trivial "free-edge" networks through the successive application of two elementary moves: "pushing" external sites into the internal and the identification of two external spins, as in Fig. 1. We will refer to the latter as amalgamation. Conversely, through the inverse, one can reduce any network to a trivial one, e.g., see Fig. 2. The first move merely changes what is called external and internal; thus it does not modify correlators.
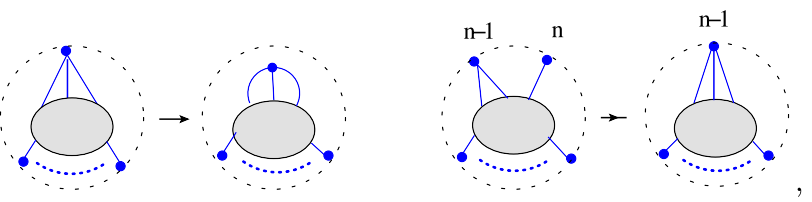

FIG. 1. Two elementary moves that yield all Ising networks.

For the second, there is a simple relation between correlators before and after amalgamation:

$$
\left\langle\sigma_{i} \sigma_{j}\right\rangle^{\mathrm{amal}}=\frac{\left\langle\sigma_{i} \sigma_{j}\right\rangle+\left\langle\sigma_{n} \sigma_{n-1} \sigma_{i} \sigma_{j}\right\rangle}{1+\left\langle\sigma_{n} \sigma_{n-1}\right\rangle},
$$

where $\langle\cdots\rangle^{\text {amal }}$ represents correlation functions of the amalgamated network [9]. While these moves act very differently on the correlators, their images in $\mathrm{OG}_{n}$ are actually identical. In both cases, we reduce the boundary sites by 1, i.e., $\mathrm{OG}_{n}$ to $\mathrm{OG}_{n-1}$. For the first case, we get the same correlator by embedding either in $\mathrm{OG}_{n}$ or $\mathrm{OG}_{n-1}$. For instance, consider from $\mathrm{OG}_{3}$ to $\mathrm{OG}_{2}$, the statement that the same $\left\langle\sigma_{1} \sigma_{2}\right\rangle$ can be obtained from either embedding leads to

$\left\langle\sigma_{1} \sigma_{2}\right\rangle=\frac{\Delta_{12}^{(2)}}{\Delta_{13}^{(2)}+\Delta_{14}^{(2)}}=\frac{\Delta_{125}^{(3)}+\Delta_{126}^{(3)}}{\Delta_{135}^{(3)}+\Delta_{136}^{(3)}+\Delta_{145}^{(3)}+\Delta_{146}^{(3)}}$,

where $\Delta_{I}^{(n)}$ are minors of $\mathrm{OG}_{n}$. The equality implies that minors of two Grassmannians are related via:

$$
\Delta_{\{I\}}^{(n-1)}=\Delta_{\{I a\}}^{(n)}+\Delta_{\{I b\}}^{(n)},
$$

where the columns $\{a, b\}(\{5,6\}$ here) correspond to that of the spin pushed into the internal. Next we consider the amalgamation of identifying sites 2 and 3 to reduce $\mathrm{OG}_{3}$ to $\mathrm{OG}_{2}$. The $3 \times 6$ matrix of $\mathrm{OG}_{3}$ are labeled as

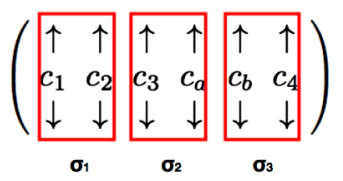

Now the extra columns $\{a, b\}$ are two adjacent columns of spin sites $\sigma_{2}$ and $\sigma_{3}$. Using the relation in Eq. (6), Eq. (3)

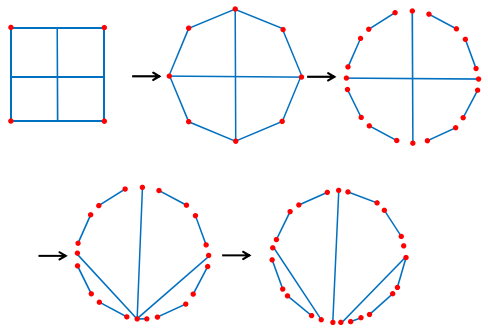

FIG. 2. Use the "inverse" of the elementary moves, an Ising network can be reduced to that of only free edges. 
again implies Eq. (8). Thus fundamental moves become identical when embedded in $\mathrm{OG}_{n}$, up to the positions of the removed columns. This is reminiscent to conformal symmetry [10]: translation and conformal boosts are drastically different, and nonlinear in general, but they are unified and linearized in twistor space.

Importantly, minors of the new network are a positive sum of those of the old one: fundamental moves preserve the positivity. Thus the positivity of general networks boils down to the property of free-edge networks, whose Grassmannian is simply the embedding of multiple $\mathrm{OG}_{\geq 0,2} \mathrm{~s}$. For ordered planar networks, it is just a block embedding,

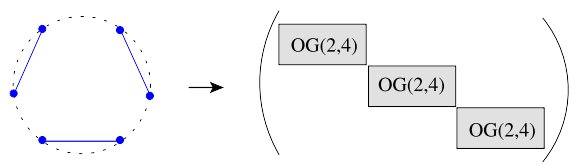

Nonvanishing minors require exactly two columns from each $\mathrm{OG}_{\geq 0,2}$, they are positive products of minors of $\mathrm{OG}_{\geq 0,2}$, which are manifestly positive. The embeddings of general planar free-edge networks are simply even permutations of the columns, away from block embeddings. Thus they all live in $\mathrm{OG}_{\geq 0, n}$, and the fundamental moves lead to the correspondence for general networks.

The structure of $\mathrm{OG}_{\geq 0, n}$ and equivalence moves.-The space of $\mathrm{OG}_{\geq 0, n}$ consists of cells, and each can be represented by an on shell diagram constructed by quartic vertices. Two diagrams are equivalent if they are related by equivalence moves [1] via a change of variables, which consists of bubble reductions and a triangle move for $\mathrm{OG}_{\geq 0, n}[2,11]$. The images of these moves in the Ising network were actually recognized long ago [12]. The change of variables of on shell diagrams then corresponds to a map between couplings of Ising networks that are dual.

There are two kinds of Ising networks whose corresponding on shell diagrams contain a bubble. First:

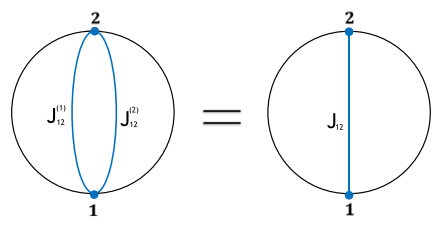

As evident from the graph, we simply have $J_{12}=J_{12}^{(1)}+J_{12}^{(2)}$. Another kind of graph is given by,

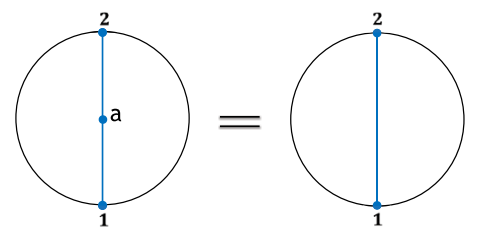

In this case, the reduction is to remove the isolated spin $a$ and to define an effective coupling via [11]

$$
\begin{aligned}
& c\left(J_{12}\right)=\frac{c\left(J_{1 a}\right) c\left(J_{2 a}\right)}{1+s\left(J_{1 a}\right) s\left(J_{2 a}\right)}, \\
& s\left(J_{12}\right)=\frac{s\left(J_{1 a}\right)+s\left(J_{2 a}\right)}{1++s\left(J_{1 a}\right) s\left(J_{2 a}\right)} .
\end{aligned}
$$

The triangle move relates two triangle on shell diagrams:

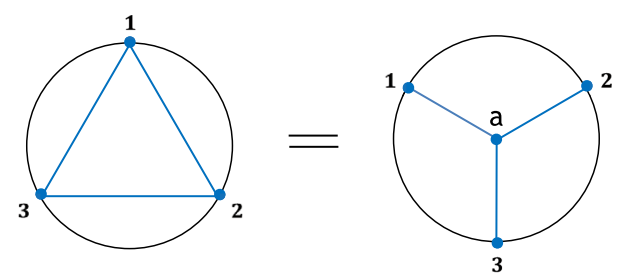

The duality transformation is given by [11]

$$
\begin{aligned}
s\left(J_{i a}\right) & =\frac{s\left(J_{i i+1}\right) c\left(J_{i+1 i+2}\right) s\left(J_{i i+2}\right)}{c\left(J_{i+1 i+2}\right)+c\left(J_{i i+1}\right) c\left(J_{i i+2}\right)}, \\
c\left(J_{i i+1}\right) & =\frac{c\left(J_{i a}\right) c\left(J_{i+1 a}\right) s\left(J_{i+2 a}\right)}{s\left(J_{i+2 a}\right)+s\left(J_{i a}\right) s\left(J_{i+1 a}\right)},
\end{aligned}
$$

for $i=1,2,3$ with $i+3:=i$ is understood.

Recursion relations through the Grassmannian.-Since the information of correlation functions of Ising networks is completely captured by $\mathrm{OG}_{\geq 0, n}$, one obtains all of the correlators by constructing the Grassmannian. This leads to new methods to compute correlators. Details of the two recursions will be illustrated for an explicit example in [13].

Recursions via duality transformations: When the equivalence moves are applied to a network with a selfsimilar structure, the map for effective couplings becomes the recursion relations of an exact RG equation type. To illustrate the idea, we consider some examples. Begin with the Sierpinski triangle:

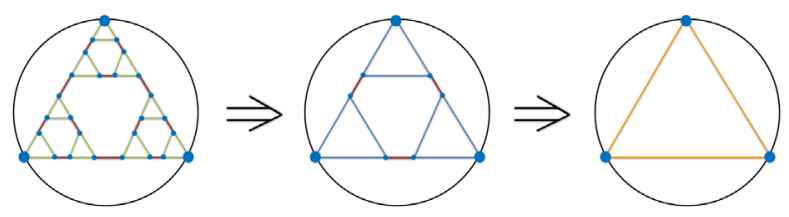

It is a system with two kinds of couplings, marked with different colors: the one connecting between triangles (called $J_{1}$ and marked with brown), whereas the rest is $J_{2}$. Using the duality transformations, we obtain the recursion relation for $J_{2}$,

$c\left(J_{2}^{\prime}\right)=\frac{c\left(J_{1}\right) c\left(J_{2}\right)^{2}}{c\left(J_{2}\right)^{2}+2\left[1-c\left(J_{2}\right)\right]\left[1+s\left(J_{1}\right)-\frac{1}{2} c\left(J_{1}\right) c\left(J_{2}\right)\right]}$.

In the limit $J_{1} \rightarrow \infty$, when we shrink all the brown edges, the above relation reduces to 


$$
c\left(J_{2}^{\prime}\right)=\frac{c\left(J_{2}\right)^{2}}{2 c\left(J_{2}\right)^{2}-3 c\left(J_{2}\right)+2}
$$

which agrees with the result in $[14,15]$. Another example we consider here is:

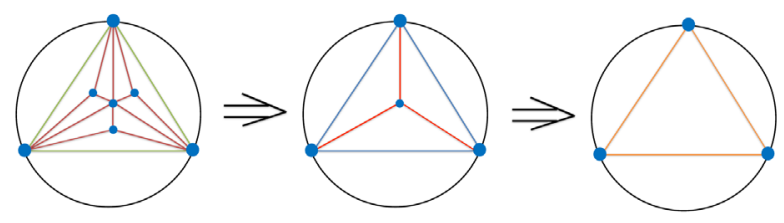

It is constructed by starting with a triangle, then it triangulates it into 3 smaller ones, and it continues with the same procedure. As shown again by the colors, the outer edges have the same coupling $J$, whereas all of the internal ones are $J_{I}$. The structure remains under the recursions. Focusing on the last step of the recursion, we have

$$
c\left(J^{\prime}\right)=\frac{1-s\left(J_{I}\right)+c(J)}{1+c(J)\left[1-s\left(J_{I}\right)\right]}
$$

We comment that the fixed points to the recursions discussed here are all simply $J=0$ or $\infty$; thus the systems do not exhibit finite temperature phase transitions.

Recursions through amalgamation: The amalgamation is agnostic to the underlying Grassmannian. Thus when recursively applying the same construction to build a large network, the complexity is constant at each iteration. For an $\mathrm{OG}_{\geq 0,2 n}$, corresponding to any network with $2 n$ boundary sites and $N_{0}$ total sites, amalgamating with itself along $n$ edges leads to

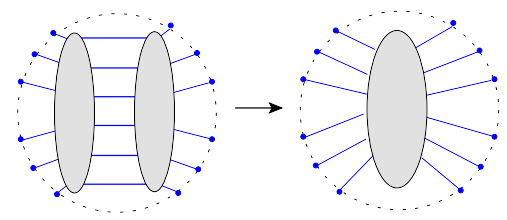

The result is a new $\mathrm{OG}_{\geq 0,2 n}$ with $2 N_{0}-n$ total sites. We note that, if the final lattice contains $N$ sites, the computation complexity scales as $\log N / N_{0}$ using the iteration method, in contrast to the linear growth for the conventional approach. As an illustration of its power, consider the iteration of the following network:

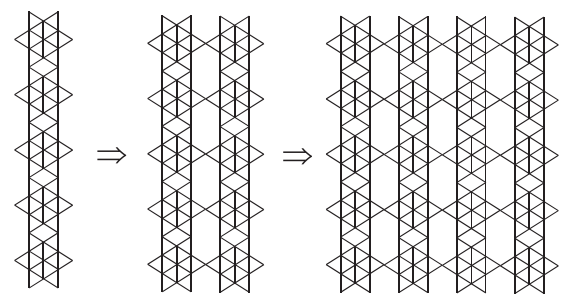

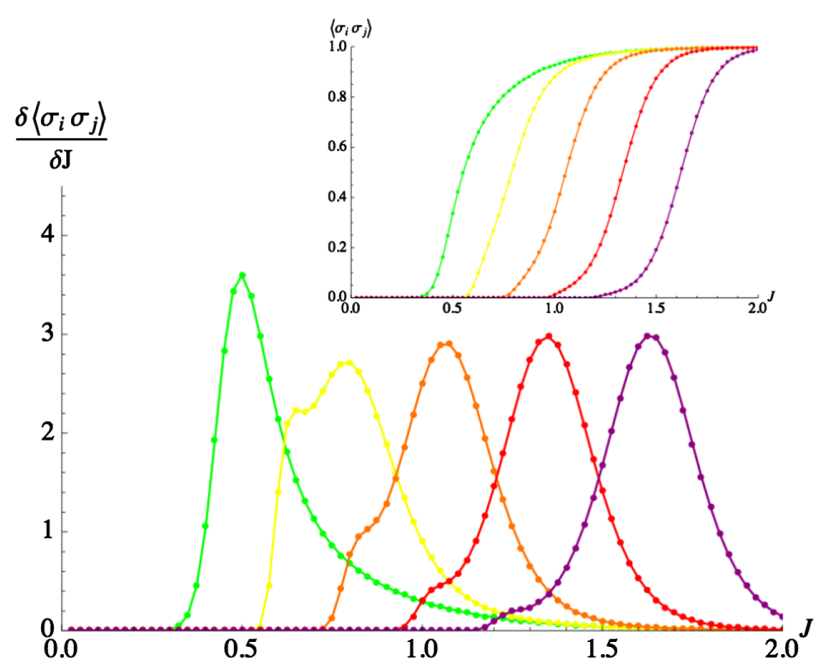

FIG. 3. The variation of the two-point function with respect to the coupling $J$ for the network in Eq. (18), with iterations 1, 5, 10, 15,20 , and 25, displayed successively to the right. The inset is the plot of the two point function itself.

where $N_{0}=57$. Straightforwardly applying the recursion to up to 25 iterations results in over $1.7 \times 10^{9}$ spin sites. The correlator, with respect to coupling $J$, as well as its tangent slope, are plotted in Fig. 3. We see that the tangent slope stabilizes under this iteration, showing no first-order phase transition.

Phase transitions: The above two recursive constructions of correlators are applicable to lattices that have a "finite ramification": those can be partitioned by removing finite vertices. For the duality based recursions, by construction the final result is dual to a simple finite lattice, while for the amalgamation recursion, the result is simply a sum of finite lattices, so one should not observe any phase transitions. This shows the lack of phase transitions for lattices with finite ramification numbers [15].

Conclusions and outlook.-We explore the correspondence between $\mathrm{OG}_{\geq 0, n}$ and $2 \mathrm{D}$ planar Ising networks, which is established via fundamental moves and the positivity of the simplest free-edge networks. The correspondence leads to duality transformations that relate networks in the same cell of the Grassmannian and the amalgamation construction for general networks. Duality transformations and amalgamation are actually applicable beyond 2D planar networks, while the positivity of freeedge networks holds even with external magnetic fields. It is of interest to explore to what extent the results in this Letter can be applied.

We thank P. Galashin and P. Pylyavskyy for bringing to our attention their fascinating work. We also like to thank Nima Arkani-Hamed for very enlightening comments. C. W. is supported by a Royal Society University Research Fellowship No. UF160350. C.-K. K. and Y.-t. H. are supported by MoST Grant No. 106-2628-M-002-012-MY3. 
[1] N. Arkani-Hamed, J. L. Bourjaily, F. Cachazo, A. B. Goncharov, A. Postnikov, and J. Trnka, Grassmannian Geometry of Scattering Amplitudes, arXiv:1212.5605.

[2] Y. T. Huang and C. Wen, ABJM amplitudes and the positive orthogonal grassmannian, J. High Energy Phys. 02 (2014) 104.

[3] N. Arkani-Hamed and J. Trnka, The amplituhedron, J. High Energy Phys. 10 (2014) 030.

[4] N. Arkani-Hamed, T-z. Huang, and Y-t. Huang (to be published); N. Arkani-Hamed, Y-t. Huang, and Shu-Heng Shao (to be published).

[5] P. Galashin and P. Pylyavskyy, Ising model and the positive orthogonal Grassmannian, arXiv:1807.03282.

[6] S. Lee, Yangian Invariant Scattering Amplitudes in Supersymmetric Chern-Simons Theory, Phys. Rev. Lett. 105, 151603 (2010).

[7] Higher-point functions can be written as products of twopoint functions, and thus the correspondence generalizes easily [5].

[8] M. Lis, The planar Ising model and total positivity, J. Stat. Phys. 166, 72 (2017).

[9] For planar networks, the four-point function can be recast as a sum of products of two-point functions, see, J. Groeneveld, R. J. Boel, and P. W. Kasteleyn, Correlation-function identities for general planar Ising systems, Physica (Amsterdam) 93A, 138 (1978).
[10] H. Elvang and Y.t. Huang, Scattering Amplitudes, arXiv: 1308.1697.

[11] Y.t. Huang, C. Wen, and D. Xie, The positive orthogonal Grassmannian and loop amplitudes of ABJM, J. Phys. A 47, 474008 (2014).

[12] The bubble reductions are the "decoration transformation" used by Naya, and the triangle move is the "start-triangle transformation" found by Onsager, see: S. Naya, On the spontaneous magnetizations of Honeycomb and Kagom Ising lattices, Prog. Theor. Phys. 11, 53 (1954); L. Onsager, Crystal statistics. I. A two-dimensional model with an order-disorder transition, Phys. Rev. 65, 117 (1944).

[13] See Supplemental Material at http://link.aps.org/supplemental/ 10.1103/PhysRevLett.121.251604 for more details about derivation of the recursion relation for Ising Model on the Sierpinski triangle using two different approaches: one we use the equivalence moves, the other we utilize the amalgamation construction.

[14] Y. Gefen, B. B. Mandelbrot, and A. Aharony, Critical Phenomena on Fractal Lattices, Phys. Rev. Lett. 45, 855 (1980).

[15] Y. Gefen, A. Aharony, Y. Shapir, and B. B. Mandelbrot, Phase transitions on fractals. II. Sierpinski gaskets, J. Phy. A 17, 435 (1984). 